\title{
The Bethe surface of liquid water
}

\author{
Michael Dingfelder* and Mitio Inokuti ${ }^{+}$
}

GSF - National Research Center for Environment and Health Institute for Radiation Protection

Ingolstaedter Landstrasse 1

D-85764 Neuherberg, Germany

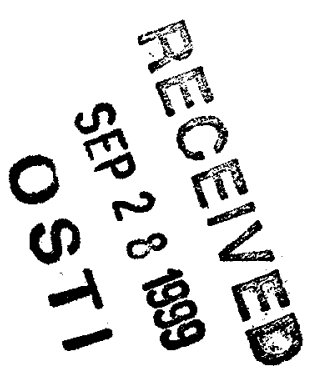

\begin{abstract}
The Bethe surface of liquid water,earlier calculated using a semi-empirical model, is compared with recent available data from IXS-experiments (inelastic X-ray scattering; Compton scattering of high energy photons) in liquid water. No alarming discrepancy is found on a global view of the Bethe surface. The extrapolation to the optical limit (viz., at zero momentum transfer) is shown and the reliability of these data is discussed in detail.
\end{abstract}

*Correspondence should be addressed to:

Dr. Michael Dingfelder

GSF-National Research Center for Environment and Health

Institute of Radiation Protection

Ingolstaeder Landstrasse 1

D-85764 Neuherberg, Germany.

Tel: (Germany) - $89-31874113$, Fax: (Germany) - $89-31873363$, email: dingfelder@gsf.de

+ Permanent address: Physics Division, Argonne National Laboratory, 9700 South Cass Avenue, Argonne, Illinois 60439-4843, U. S. A., email: inokuti@anl.gov 


\section{DISCLAIMER}

This report was prepared as an account of work sponsored by an agency of the United States Government. Neither the United States Government nor any agency thereof, nor any of their employees, make any warranty, express or implied, or assumes any legal liability or responsibility for the accuracy, completeness, or usefulness of any information, apparatus, product, or process disclosed, or represents that its use would not infringe privately owned rights. Reference herein to any specific commercial product, process, or service by trade name, trademark, manufacturer, or otherwise does not necessarily constitute or imply its endorsement, recommendation, or favoring by the United States Government or any agency thereof. The views and opinions of authors expressed herein do not necessarily state or reflect those of the United States Government or any agency thereof. 


\title{
DISCLAIMER
}

\author{
Portions of this document may be illegible \\ in electronic image products. Images are \\ produced from the best available original \\ document.
}




\section{Introduction}

The dielectric-response function $\epsilon(E, K)$ as a function of the energy transfer $E$ and the momentum transfer $q=\hbar K$ is the central, nontrivial quantity governing many properties of a material, including cross sections for inelastic scattering of fast charged particles. Dingfelder et al. [1] constructed a complete set of data for $\epsilon(E, K)$ semiempirically, and used them to determine cross-sections for electron inelastic scattering suitable as input to track-structure calculations and other applications.

After the completion of the semi-empirical determination [1] of the dielectric-response function, we became aware of the recent measurements by the Sendai group $[2,3]$. Workers of this group studied the Compton scattering of high-energy photons of synchrotron radiation in liquid water (and in other condensed-phase substances too). The cross section for the Compton scattering of a photon by electrons in atoms, molecules, or condensed matter is expressible as the product of the Klein-Nishina cross section (which applies to a free electron at rest) and a factor that accounts for the influence of the binding of those electrons when their binding energies are much smaller than the photon energy and when the momentum transfer is not very small. This factor is in essence the generalized oscillator-strength spectrum, or the dynamic structure factor $S(q, E)$. Therefore, it was sensible for those workers to present results in the form of the Bethe surface, viz., a plot of the energy loss function $\eta_{2}(E, K)=\operatorname{Im}[-1 / \epsilon(E, K)]$ over the plane indicating energy transfer $E$ and momentum transfer $\hbar K$.

The method of the Sendai group is particularly effective for the region near the Bethe ridge [4], viz., the peak around the line $E=(\hbar K)^{2} /(2 m)$, or $E / R y=\left(K a_{0}\right)^{2}$, corresponding to the transfer of energy and momentum to a free electron, where $R y$ is the Rydberg energy $13.606 \mathrm{eV}$ and $a_{0}$ is the Bohr radius $0.529177 \times 10^{-10} \mathrm{~m}$. In this sense, the method is complementary to the optical measurements of Heller et al. [5], which was the main source of data of Dingfelder et al. [1], who introduced the $\mathrm{K}$-dependence for the continuum through an impulse approximation.

The method of the Sendai group is suitable for condensed matter because it uses photons of such high energies $(7271,7358$, and $7503 \mathrm{eV})$, far above the K-shell threshold of oxygen and therefore little absorbed by water. In this sense it is also complementary 
to electron energy-loss measurements, which were indeed successfully carried out for the Bethe-surface determination of gaseous molecules $[6,7]$ but are difficult for condensed matter because of the influence of multiple collisions especially for appreciable momentum-transfer values.

\section{Comparison of data}

The comparison of data is done in three steps. First, the Bethe surface, i. e., the plot of $\eta_{2}(E, K)$ versus $E$ and $K$, is plotted for both approaches. Second, the extrapolation of the Sendai data for $K \rightarrow 0$ is compared with optical data and other semi-empirical approaches. Finally, some remarks on possible error sources are given.

The energy loss function $\eta_{2}(E, K)$ from the Sendai data is obtained as follows: All possible data sources from $[2,3]$ are scanned and values for $\eta_{2}(E, K)$ are extracted at various $(E, K)$-points. Because data are available only in limited $(E, K)$-ranges, gridding and interpolation and extrapolation methods, respectively, are used to produce data on a regular mesh over a wider range of $E$ and $K$ values $(0 \leq K \leq 3.6$ a.u., $0 \leq E \leq 150 \mathrm{eV}$ ). The obtained Bethe surface is displayed in Fig. 1, left panel. The $\mathrm{x}$-axis represents the energy transfer $E$ in $\mathrm{eV}$, the y-axis $\ln \left(K a_{0}\right)^{2}$, while the vertical axis represents the energy loss function $\eta_{2}(E, K)$.

The right panel of Fig. 1 shows the calculated Bethe surface from the semi-empirical model [1]. Here the imaginary part $\epsilon_{2}(E, K)$ of the dielectric response function $\epsilon(E, K)$ was modeled by a linear superposition of Drude-like functions in the optical limit, i.e., $K=0$, and fitted to optical reflectance data [5]. The real part $\epsilon_{1}(E, K)$ was calculated using the Kramers-Kronig relation. The $K$-dependence for the continuum in the sense of the impulse approximation is introduced to obtain the full energy transfer and momentum transfer dependency of the dielectric response function.

Comparing both sets of data, one sees no substantial difference. Both data sets are constrained by the Bethe sum rule, which is valid at each $K$-value. At low $K$ values, the energy loss function is peaked at around $23 \mathrm{eV}$. The Bethe ridge, i.e., the peak at around $E=(\hbar K)^{2} / 2 m$ is well developed, sharp, and can clearly be seen in the semi-empirical approach. In contrast, the Bethe ridge in the Sendai data is much 
more broader. In other words, the values away from the maximum decrease much faster, the absolute values are much smaller. At lower momentum transfer the ridge is located at lower energies, compared to the model; it remains more or less at the same energy for increasing momentum transfer. At higher momentum and energy transfer, the ridge is located on the line corresponding to the free-electron energy-momentum relation, as expected. The broader ridge might be closer to reality than the sharply peaked theoretical curve, but probably will not greatly affect the single differential cross section values, $d \Sigma / d E$, which are obtained by integrating $\eta_{2}(E, K)$ over $\mathrm{K}$.

The measured energy loss function for several small and decreasing $K$ values (down to $K=0.19$ a.u.) shows near convergence for $\eta_{2}(E, K)$ for $K \rightarrow 0$, and an extrapolation to $K=0$ values is possible. However, the reliability of the measurements at low $K-$ values is questionable because of the low signal-to-noise ratio in the experiment. Also, the normalization at very low $K$-values was done in a different way: Instead of using the Bethe sum rule $\int E \eta_{2}(E) d E$, which gives too much weight on the large energy loss region due to parasitic scatterings, the IXS spectra were normalized by using the theoretically calculated static structure factor $S(q)=\int S(q, E) d E[3]$.

Nevertheless, this extrapolation is shown in Fig. 2 and compared to the optical reflectance data by Heller et al. [5] and to two semi-empirical calculations of the energy loss function by Dingfelder et al. [1] and by the Oak Ridge group [8]. At small energies the new measurements are consistent with the earlier optical data as well as the semiempirical model. The absolute values of the Sendai data are slightly smaller, the maximum, compared to $21.5 \mathrm{eV}$ for the optical data, is shifted to about $23 \mathrm{eV}$, and is lower than the semi-empirical model. At higher energies, where no optical data are available $(E>26.5 \mathrm{eV})$, the Sendai data show a plateau (25 to $35 \mathrm{eV}$ ) and overestimate the semi-empirical model clearly. In this energy region only the $\left(2 a_{1}\right)$-ionisation shell $\left(E_{\text {ion }}=32.2 \mathrm{eV}[1]\right)$ is located. This subshell alone cannot be responsible for such a plateau. Also, there is no evidence for a collective or plasmon excitation in liquid water [9], favored by the Oak Ridge group [8], but questioned by Dingfelder et al. [1]. Therefore, especially in this higher energy region, the Sendai data for small momentum transfer values have to be questioned in view of the above mentioned experimental problems. New and more precise measurement are desirable. 
At small energies the new measurement reproduces the optical data, while for larger energy transfers $(E>30 \mathrm{eV})$ the Sendai data are higher and decrease slower than the semi-empirical model.

Another possible source for an inaccuracy of the Sendai-data concerns the relation between energy transfer $E$, scattering angle $\theta$ and momentum transfer $q=\hbar K$. The Sendai group uses an approximate relation, $q=2(\hbar \omega / c) \sin (\theta / 2)$, where $\hbar \omega$ is the energy of the incident radiation to determine the momentum transfer. The rigorous relation, $q^{2}=\left|\vec{p}-\vec{p}^{\prime}\right|^{2}=p^{2}+p^{\prime 2}-2 p p^{\prime} \cos (\theta)$, where $\vec{p}$ is the momentum before scattering and $\vec{p}^{\prime}$ that one after scattering, $p=\hbar \omega / c, p^{\prime}=\hbar \omega^{\prime} / c=(\hbar \omega-E) / c$ leads to:

$$
q^{2}=(\hbar \omega / c)^{2}\left[\xi^{2}+4(1-\xi) \sin ^{2}(\theta / 2)\right],
$$

where $\xi=E / \hbar \omega$. If the impulse approximation is to be used, $\xi$ should be small. Indeed, for $\xi \rightarrow 0$, Eq. (1) reduces to $q^{2}=4(\hbar \omega / c)^{2} \sin ^{2}(\theta / 2)$. Also, the minimum momentum transfer, which occurs at $\theta=0$, is $q_{\min }=E / c$. Expressed in atomic units, $\left(K a_{0}\right)_{\min }=\alpha E / 2 R y$, with $\alpha=e^{2} / \hbar c=1 / 137$ the fine structure constant, is indeed a small number for small energy transfers $E$. Fig. 3 shows the function in the square brackets of Eq. (1), $g(\xi, \theta)$, as a function of $\xi$ in the range from 0 to 1 and of the scattering angle $\theta$ in the range from 0 to $\pi$. One sees that there are no big changes in the relation of $\theta$ to $q$ for small $\xi$ values, while the relation changes smoothly at higher $\xi$ values. Therefore, for small $\xi$ values the use of the impulse approximation may be valid.

\section{Conclusion}

Comparison of the Sendai measurements with the semi-empirical determination indicates the following. First, one sees no alarming discrepancy on a global view of the Bethe surface, in part because the two sets of data have been constrained by the Bethe sum rule. Second, the Sendai data are probably unreliable near the optical limit, $K \rightarrow 0$, because of the low signal-to-noise ratio. Third, the shape of Bethe ridge given by the Sendai measurements is broader than obtained through the impulse approximation, and is probably closer to reality. However, it is unlikely that this difference is 
affect the cross sections for energy transfer from fast charged particles obtained through the integration over $K$. Consequently, we continue to use in the present work the data for $\eta_{2}(E, K)$ given by the semi-empirical determination. Further experimental data, especially at low- $\mathrm{K}$ values are desirable, for an improved determination of the Bethe surface.

\section{Acknowledgements}

The work is supported by the European Community under Contract No. FI4P-CT950011 "Biophysical Models for the Induction of Cancer by Radiation" and in part by the U. S. Department of Energy under Contract No. W-31-109-Eng-38. 


\section{References}

[1] Dingfelder M, Hantke D, Inokuti M, Paretzke HG (1998) Electron inelasticscattering cross sections in liquid water. Radiat Phys Chem 53:1-18

[2] Watanabe N, Hayashi H, Udagawa Y (1997) Bethe Surface of Liquid Water Determined by Inelastic X-Ray Scattering Spectroscopy and Electron Correlation Effects. Bull Chem Soc Jpn 70:719-726

[3] Hayashi H, Watanabe N, Udagawa Y, Kao CC (1998) Optical spectra of liquid water in vacuum uv region by means of inelastic $x$-ray scattering spectroscopy. $J$ Chem Phys 108:823-825

[4] Inokuti M (1971) Inelastic Collisions of Fast Charged Particles with Atoms and Molecules - The Bethe Theory Revisited. Rev Mod Phys 43:297-347

[5] Heller Jr JM, Hamm RN, Birkhoff RD, Painter RL (1974) Collective oscillation in liquid water. J Chem Phys 60:3483-3486

[6] Bonham RA (1990). Scanning Microscopy Suppl 4:1-15

[7] Chetioui A, Herve Du Penhoat MA, Fayard B, Champion C, L'Hoir A, Touati A, Abel F, Politis MF, Despiney-Bailly I, Sabatier L (1998) K-shell ionisation and biological effects. In: Aumayr F, Winter HP (eds) Photonic, Electronic and Atomic Collisions. World Scientific, Singapore, pp 519-524

[8] Ritchie RH, Hamm RN., Turner JE, Wright HA, Bolch WE (1991) Radiation Interactions and Energy Transport in the Condensed Phase. In: Glass WA, Varma MN (eds) Physical and Chemical Mechanisms in Molecular Radiation Biology. Plenum Press, New York 1991, pp 99-133

[9] La Verne JA, Mozumder A (1993) Concerning plasmon excitation in liquid water. Radiat Res 133:282-288 


\section{Fiures}

Figure 1: Bethe-surface: Energy loss function $\eta_{2}(E, K)$ versus energy transfer $E$ (in $\mathrm{eV})$ and $\ln \left(K a_{0}\right)^{2}$, where $\hbar K$ is the momentum transfer. Left panel: Sendai-data $[2,3]$. Right panel: Semi-empirical model [1].

Figure 2: Energy loss function $\eta_{2}(E, K)$ in the optical limit $(K=0)$ versus energy transfer $E$ (in $\mathrm{eV}$ ). Sendai data [3]: o; Heller data[5]: $\square$; Dingfelders calculation [1]: solid line; Oak Ridge calculation [1]: dashed line.

Figure 3: Relation between momentum transfer $q$, scattering angle $\theta$ and energy transfer $E: q^{2}=(\hbar \omega / c)^{2} g(\theta, \xi)$, with $\xi=E / \hbar \omega . g(\theta, \xi)$ is plotted as a function of $\theta$ and $\xi$. 
Fig. 1 a

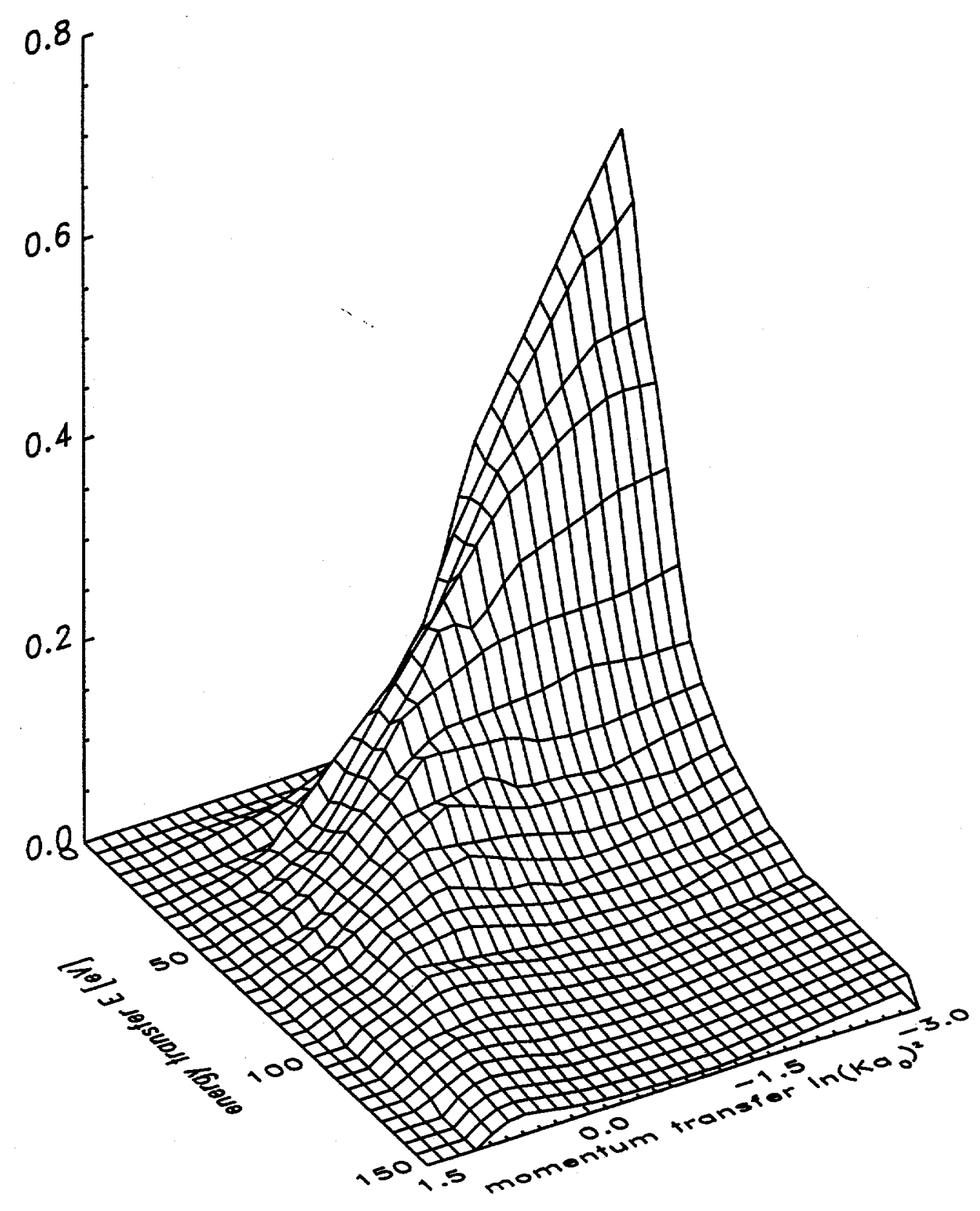


Fig. 1 b

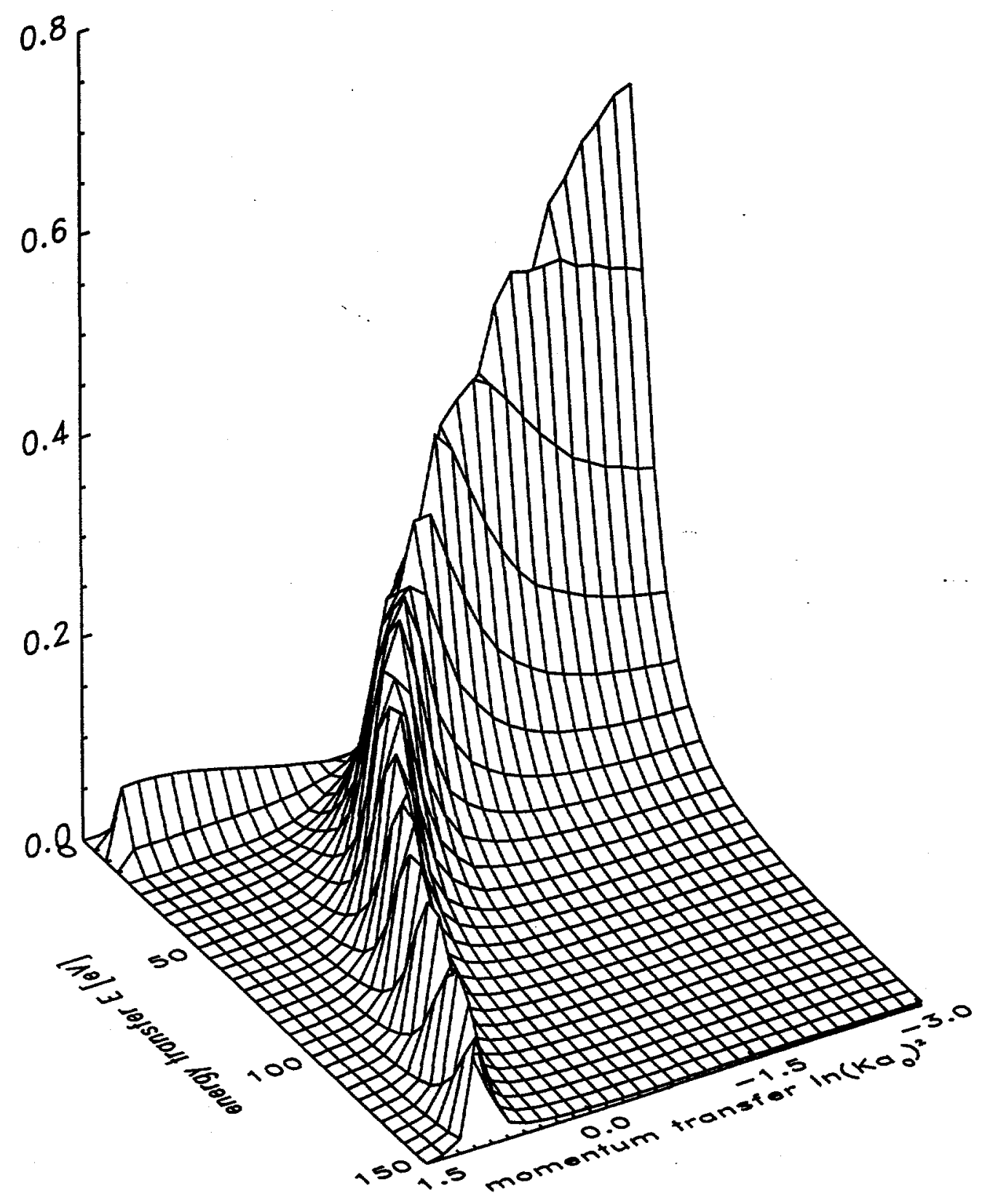


Fig. 2

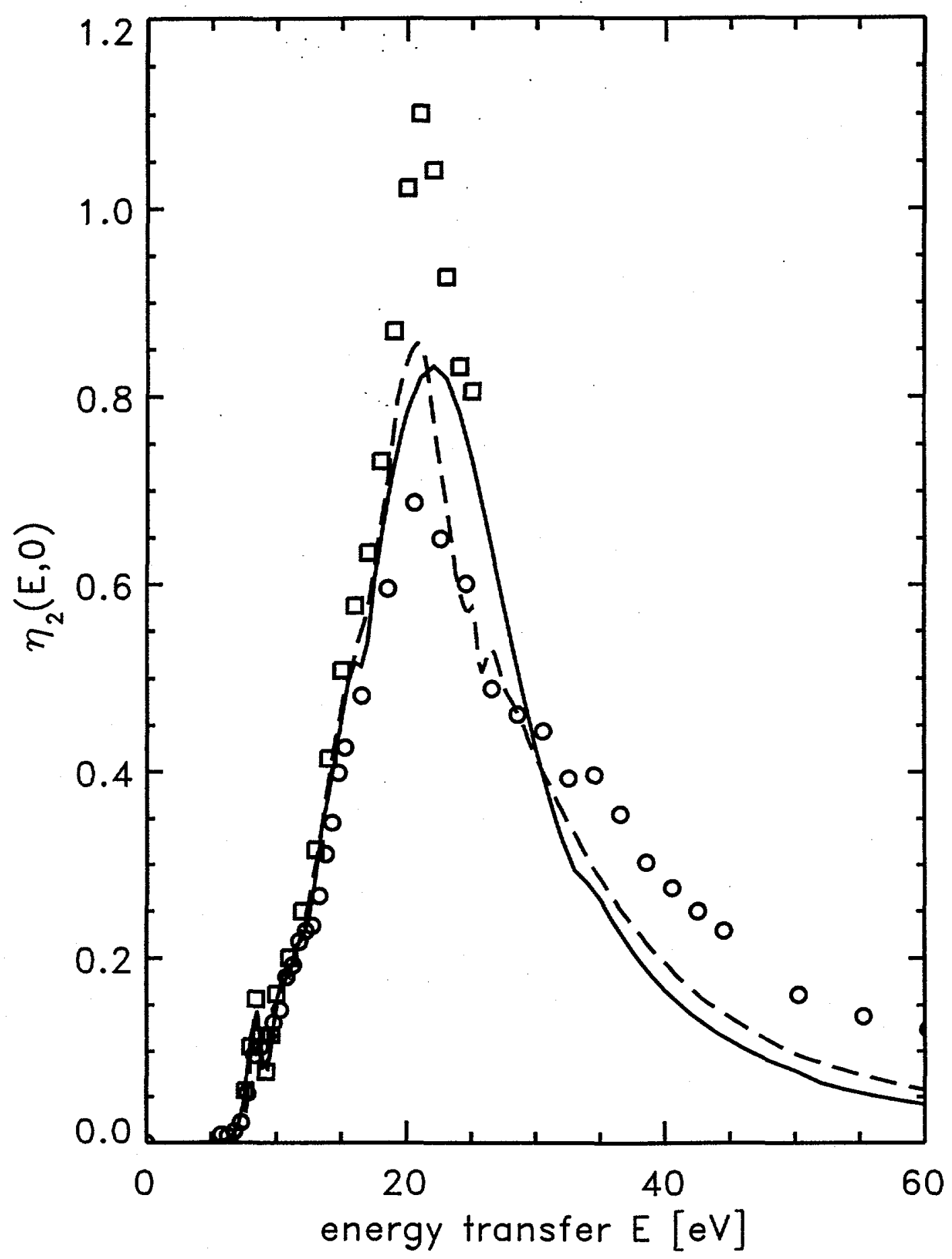


Fig. 3

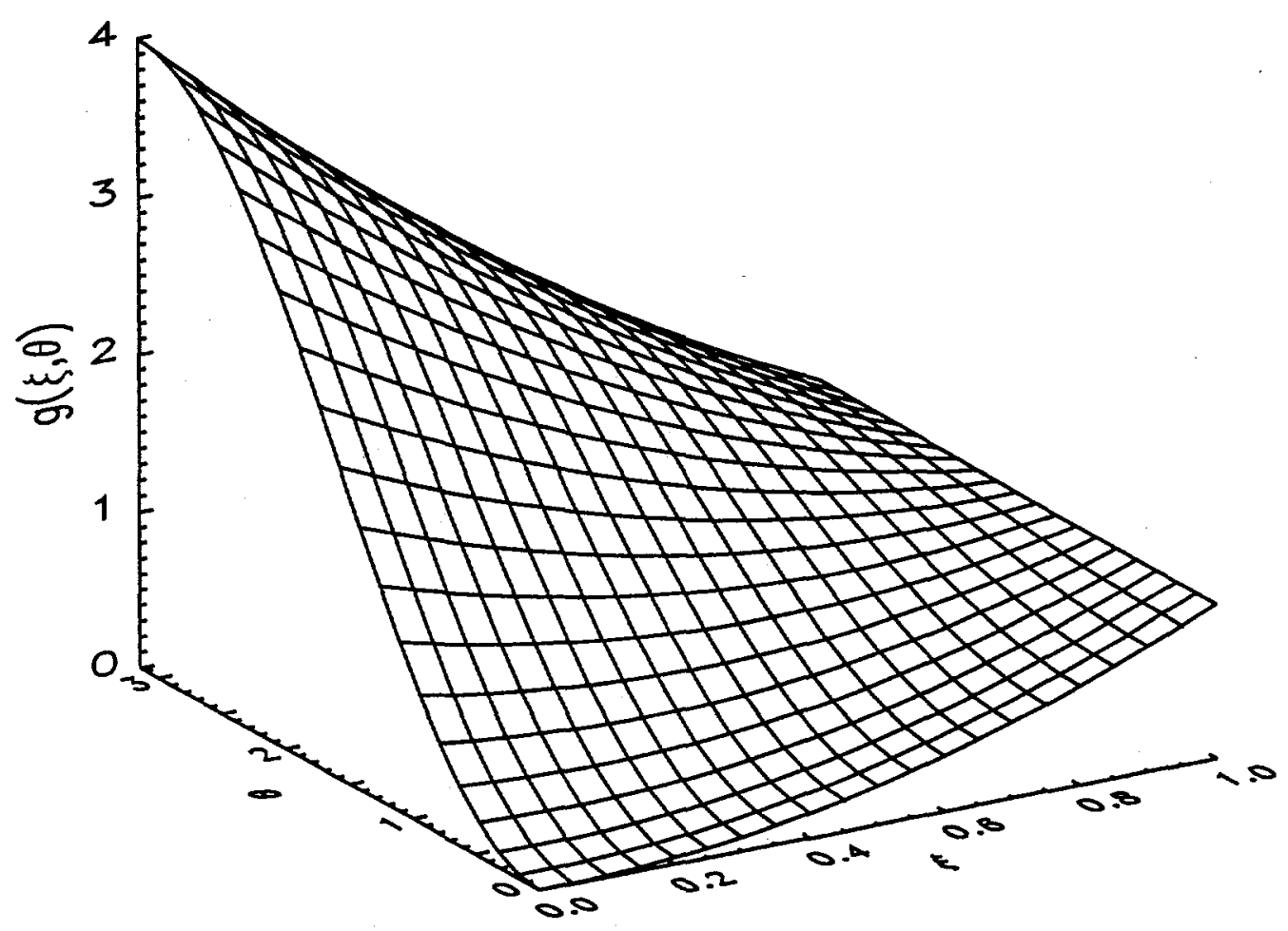

